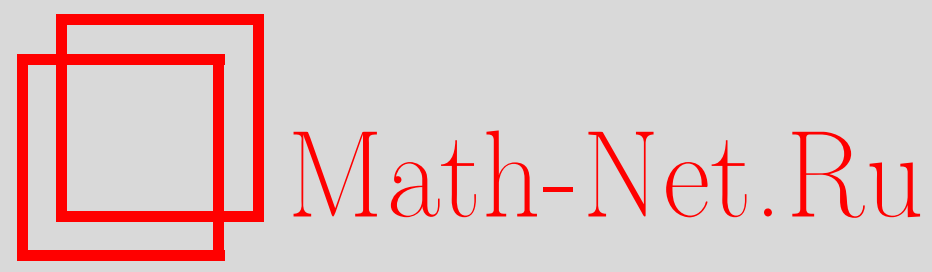

А. М. Романов, О комбинаторных кодах Грея с расстоянием 3, Дискрет. матем., 2009, том 21, выпуск 3, 73-78

DOI: https://doi.org/10.4213/dm1062

Использование Общероссийского математического портала Math-Net.Ru подразумевает, что вы прочитали и согласны с пользовательским соглашением http://www.mathnet.ru/rus/agreement

Параметры загрузки:

IP: 54.162 .85 .209

26 апреля 2023 г., 13:44:57 


\title{
О комбинаторных кодах Грея с расстоянием 3
}

\author{
(ㄷ) 2009 г. А. М. Романов
}

\begin{abstract}
Предложена конструкция циклических двоичных комбинаторных кодов Грея с расстоянием 3 размерности $n=2^{k}-1$, где $k=3,4, \ldots$ Дан метод построения гамильтоновых циклов в графах минимальных расстояний двоичных кодов Хемминга. Для всех допустимых длин $n \geqslant 15$ приводятся нелинейные совершенные двоичные коды, графы минимальных расстояний которых содержат гамильтонов цикл.

Работа выполнена при поддержке Российского фонда фундаментальных исследований, проект 05-01-00364.
\end{abstract}

\section{1. Введение}

Пусть $\mathbf{F}_{2}^{n}-$ множество всех двоичных векторов длины $n$ над полем Галуа $\mathbf{F}_{2}=\{0,1\}$. Расстоянием Хемминга $d(\mathbf{x}, \mathbf{y})$ между векторами $\mathbf{x}, \mathbf{y} \in \mathbf{F}_{2}^{n}$ называется число координат, в которых векторы $\mathbf{x}$ и $\mathbf{y}$ различаются. Биективное отображение множества $\mathbf{F}_{2}^{n}$ на множество $\left\{1,2, \ldots, 2^{n}\right\}$ определяет линейный порядок на $\mathbf{F}_{2}^{n}$. Линейно упорядоченное множество $\mathbf{F}_{2}^{n}$ называется двоичным комбинаторным кодом Грея размерности $n$ с расстоянием $d$, если расстояние Хемминга между двумя произвольными последовательными векторами равно $d$. При $d=1$ комбинаторный двоичный код Грея с расстоянием $d$ является классическим кодом Грея. Термин “комбинаторные коды Грея” используется для широкого круга задач, в которых комбинаторные объекты (например, $k$-элементные подмножества $n$-элементного множества или перестановки, действующие на множестве из $n$ элементов) линейно упорядочены так, что произвольные последовательные элементы отличаются некоторым фиксированным образом [1]. При этом слово “комбинаторный” часто опускается. Мы также далее вместо комбинаторный код Грея будем писать просто код Грея. Известно, что двоичные коды Грея с расстоянием $d$ существуют только при нечетных $d$ (см. [2]). Код Грея называется циклическим, если расстояние между первым и последним векторами также равно $d$. Граф называется $n$-мерным булевым кубом, если его множество вершин совпадает с $\mathbf{F}_{2}^{n}$ и две вершины графа являются смежными тогда и только тогда, когда расстояние Хемминга между ними равно 1. Гамильтоновым циклом называется простой цикл, содержащий все вершины графа ровно по одному разу. Граф, содержащий гамильтонов цикл, называется гамильтоновым. Гамильтонов цикл в $n$-мерном булевом кубе соответствует коду Грея размерности $n$ с расстоянием $d=1$. Остается нерешенной гипотеза Ласло Ловаша, в которой утверждается, что из вершинной транзитивности связного графа следует существование в нем гамильтонова цикла [1].

В данной работе предложена конструкция циклических двоичных кодов Грея с расстоянием 3 размерности $n=2^{k}-1$, где $k=3,4, \ldots$ В основе конструкции лежат разбиения 
множества $\mathbf{F}_{2}^{n}$ на смежные классы, образованные кодами Хемминга. В работе дана конструкция гамильтоновых циклов в графах минимальных расстояний двоичных кодов Хемминга. Остается открытым вопрос о гамильтоновости графов минимальных расстояний совершенных кодов. Для всех допустимых длин $n \geqslant 15$ приводятся нелинейные совершенные двоичные коды, графы минимальных расстояний которых содержат гамильтонов цикл. Предложенные нелинейные совершенные коды построены при помощи свитчингов и uv-конструкции.

Следует заметить, что гамильтоновы циклы в графах минимальных расстояний совершенных двоичных кодов соответствуют кодам, которые принято называть змеей в ящике (то есть таким линейно упорядоченным подмножествам векторов из $\mathbf{F}_{2}^{n}$, что расстояние между двумя произвольными последовательными векторами из этого подмножества равно $d_{1}$ и минимально возможное расстояние между двумя различными не являющимися последовательными векторами из подмножества равно $d_{2}$; в нашем случае $d_{1}=d_{2}=3$ ).

\section{2. Коды Хемминга}

Множество $\mathbf{F}_{2}^{n}$ образует линейное пространство размерности $n$ над полем $\mathbf{F}_{2}$. Векторы из множества $\mathbf{F}_{2}^{n}$ рассматриваются как двоичные слова длины $n$. Произвольное подмножество $\mathbf{C} \subseteq \mathbf{F}_{2}^{n}$ называется двоичным кодом длины $n$. Векторы, принадлежащие коду, называются кодовыми словами. Минимально возможное расстояние $d$ между двумя различными кодовыми словами называется минимальным расстоянием кода. Код $\mathbf{C} \subseteq \mathbf{F}_{2}^{n}$ называется совершенным двоичным кодом с минимальным расстоянием $d=2 \rho+1$, если для каждого вектора $\mathbf{x} \in \mathbf{F}_{2}^{n}$ существует единственное кодовое слово $\mathbf{c} \in \mathbf{C}$ такое, что $d(\mathbf{x}, \mathbf{c}) \leqslant \rho$. Код называется линейным, если его слова образуют линейное подпространство в $\mathbf{F}_{2}^{n}$. Линейные совершенные коды с минимальным расстоянием $d=3$ называются кодами Хемминга. Коды $\mathbf{C}_{1}$ и $\mathbf{C}_{2}$ называются эквивалентными, если существует вектор $\mathbf{x} \in \mathbf{F}_{2}^{n}$ и перестановка $\pi$, действующая на множестве координат, такие, что $\mathbf{C}_{1}=\left\{\pi(\mathbf{c})+\mathbf{x}: \mathbf{c} \in \mathbf{C}_{2}\right\}$. С точностью до эквивалентности существует единственный двоичный код Хемминга длины $n$.

Длина кода $n$, мощность $M$, минимальное расстояние $d$ называются параметрами кода. Известно, что совершенные двоичные коды с параметрами кодов Хемминга существуют только при $n=2^{k}-1, k=2,3, \ldots$ (см. [3]). Нелинейные совершенные двоичные коды имеют параметры кодов Хемминга и существуют при $n \geqslant 15$. Далее мы будем предполагать, что нулевой вектор всегда принадлежит коду.

Пусть C - совершенный код с параметрами кода Хемминга. Графом минимальных расстояний кода $\mathbf{C}$ называется граф $G(\mathbf{C})$, множество вершин которого совпадает с $\mathbf{C}$ и вершины $\mathbf{x}, \mathbf{y} \in G(\mathbf{C})$ являются смежными тогда и только тогда, когда $d(\mathbf{x}, \mathbf{y})=3$.

Через $p(\mathbf{x})$ обозначим функцию четности, то есть

$$
p(\mathbf{x})=p\left(x_{1}, x_{2}, \ldots, x_{n}\right)=x_{1}+x_{2}+\ldots+x_{n} \quad(\bmod 2),
$$

где $\mathbf{x}=\left(x_{1}, x_{2}, \ldots, x_{n}\right) \in \mathbf{F}_{2}^{n}$.

При $k=2,3, \ldots$ положим

$$
\mathbf{R}_{k}=\left\{(\mathbf{u}|p(\mathbf{u})| \mathbf{u}): \mathbf{u} \in \mathbf{F}_{2}^{(n-1) / 2},(n-1) / 2=2^{k-1}-1\right\},
$$

вертикальная черта $(\cdot \mid \cdot)$ обозначает конкатенацию.

В силу конструкции минимально возможное расстояние между двумя различными векторами из множества $\mathbf{R}_{k}$ равно 3 . Через $G\left(\mathbf{R}_{k}\right)$ обозначим граф минимальных расстояний множества $\mathbf{R}_{k}$. 
Предложение 1. При $k=2,3, \ldots$ граф $G\left(\mathbf{R}_{k}\right)$ изоморфен булеву кубу размерности $(n-1) / 2=2^{k-1}-1$.

Доказательство. Отображение $(\mathbf{u}|p(\mathbf{u})| \mathbf{u}) \rightarrow \mathbf{u}$ является биективным и сохраняет отношение смежности. Предложение 1 доказано.

Через $\mathbf{H}_{k}$ обозначим двоичный код Хемминга длины $n=2^{k}-1, k=2,3, \ldots$

Предложение 2. При $k=2,3, \ldots$ в графе минимальных расстояний двоччного кода Хемминга $\mathbf{H}_{k}$ длины $n=2^{k}-1$ существует остовный подграф, изоморфньй булеву кубу размерности $n-k$.

Доказательство. Предложение доказывается индукцией по $k$. При $k=2$ справедливость предложение очевидна. Покажем, что из индуктивного предположения о справедливости предложения при $k-1$ следует его справедливость при $k$. Рассмотрим uv-конструкцию кодов Хемминга $[4,5]$ ( uv-конструкция - короткое название хорошо известной $(\mathbf{u} \mid \mathbf{u}+\mathbf{v})$ конструкции [3]). Для кода Хемминга $\mathbf{H}_{k}$ справедливо представление

$$
\mathbf{H}_{k}=\left\{(\mathbf{u}|p(\mathbf{u})| \mathbf{u}+\mathbf{v}): \mathbf{u} \in \mathbf{F}_{2}^{(n-1) / 2}, \mathbf{v} \in \mathbf{H}_{k-1},(n-1) / 2=2^{k-1}-1\right\} .
$$

С учетом введенных выше обозначений запишем формулу (1) в виде

$$
\mathbf{H}_{k}=\bigcup_{\mathbf{v} \in \mathbf{H}_{k-1}}\left(\mathbf{R}_{k}+(\mathbf{0} \mid \mathbf{v})\right),
$$

где 0 - нулевой вектор длины $(n+1) / 2=2^{k-1}$. Из формулы $(2)$, индуктивного предположения и предложения 1 следует справедливость предложения 2.

Теорема 1. При $k=3,4, \ldots$ граф минимальных расстояний двоччного кода Хемминга $\mathbf{H}_{k}$ является гамильтоновым. Более того, через любые две смежные вершины этого графа проходит гамильтонов циикл.

Доказательство. В силу предложения 2 в графе $G\left(\mathbf{H}_{k}\right)$ содержится остовный подграф, изоморфный булеву кубу. Известно, что булев куб содержит гамильтонов цикл и через любые две смежные вершины булева куба проходит гамильтонов цикл. Теорема 1 доказана.

\section{3. Коды Грея}

При $i=1,2, \ldots, n$ через $\mathbf{e}_{i}$ обозначим двоичный вектор, в котором $i$-я координата равна 1 , а остальные координаты равны 0 , при $i=0$ вектор $\mathbf{e}_{i}$ положим равным нулевыму вектору. Весом $w(\mathbf{x})$ вектора $\mathbf{x} \in \mathbf{F}_{2}^{n}$ называется число ненулевых координат в $\mathbf{x}$. Носителем вектора $\mathbf{x}=\left(x_{1}, x_{2}, \ldots, x_{n}\right) \in \mathbf{F}_{2}^{n}$ называется множество $[\mathbf{x}]=\left\{i: x_{i}=1\right\}$. Через $|[\mathbf{x}]|$ обозначим мощность множества $[\mathbf{x}]$. Очевидно, что $w(\mathbf{x})=|[\mathbf{x}]|$.

Предложение 3. Пусть векторы $\mathbf{c}, \mathbf{c}^{\prime} \in \mathbf{H}_{k}+\mathbf{e}_{i}, \mathbf{c} \neq \mathbf{c}^{\prime}, i \in\{0,1, \ldots, n\}, w(\mathbf{c})=w\left(\mathbf{c}^{\prime}\right)=3$. Тогда справедливо неравенство $\left|[\mathbf{c}] \cap\left[\mathbf{c}^{\prime}\right]\right| \leqslant 1$.

Доказательство. Допустим, что $[\mathbf{c}] \cap\left[\mathbf{c}^{\prime}\right] \neq \varnothing$. Тогда при $i=0$ множество $\mathbf{H}_{k}+\mathbf{e}_{i}$ является кодом Хемминга $\mathbf{H}_{k}$, содержащим нулевой вектор. Известно, что семейство $\left\{[\mathbf{v}]: \mathbf{v} \in \mathbf{H}_{k}, w(\mathbf{v})=3\right\}$ подмножеств множества $\{1,2, \ldots, n\}$ является системой троек Штейнера. Непосредственно из определения системы троек Штейнера следует, что при 
$\mathbf{c} \neq \mathbf{c}^{\prime}, w(\mathbf{c})=w\left(\mathbf{c}^{\prime}\right)=3$ справедливо неравенство $\left|[\mathbf{c}] \cap\left[\mathbf{c}^{\prime}\right]\right| \leqslant 1$. При $i \in\{1,2, \ldots, n\}$ в силу транзитивности группы автоморфизмов кода $\mathbf{H}_{k}$ без ограничения общности будем считать, что $i \leqslant(n-1) / 2$ и $\{i+(n+1) / 2\} \in[\mathbf{c}] \cap\left[\mathbf{c}^{\prime}\right]$. Тогда из (1) следует, что все носители векторов веса 3 из смежного класса $\mathbf{H}_{k}+\mathbf{e}_{i}$ с единичной $i+(n+1) / 2$ координатой имеют вид $\{1, i+(n+1) / 2,1+i+(n+1) / 2\},\{2, i+(n+1) / 2,2+i+(n+1) / 2\}, \ldots$, $\{(n-1) / 2, i+(n+1) / 2,(n-1) / 2+i+(n+1) / 2\}$. Единственным общим элементом в приведенных выше подмножествах является координата $i+(n+1) / 2$. Предложение 3 доказано.

Предложение 4. При $i, j \in\left\{0,1, \ldots, n=2^{k}-1\right\}$ для любого вектора $\mathbf{c} \in \mathbf{H}_{k}+\mathbf{e}_{i}$, $w(\mathbf{c})=3$, существуют векторы $\mathbf{c}^{\prime} \in \mathbf{H}_{k}+\mathbf{e}_{j} u \mathbf{v} \in \mathbf{H}_{k-1}, w\left(\mathbf{c}^{\prime}\right)=w(\mathbf{v})=3$, пакие, что

$$
d\left(\mathbf{c},\left(\mathbf{c}^{\prime}+(\mathbf{0} \mid \mathbf{v})\right)\right)=3,
$$

где $\mathbf{0}$ - нулевой вектор длины $(n+1) / 2=2^{k-1}$.

Доказательство. Координатное множество $\{1,2, \ldots, n\}$ разобьем на два подмножества $X, Y$. Положим $X=\{i: 1 \leqslant i \leqslant(n+1) / 2\}, Y=\{i:(n+1) / 2<i \leqslant n\}$. Допустим, что $i, j \in X$ и вектор $\mathbf{c} \in \mathbf{H}_{k}+\mathbf{e}_{i}$ таков, что мощность $|[\mathbf{c}] \cap X|=1$. Считаем, что координатное множество кода $\mathbf{H}_{k-1}$ имеет вид $\left\{1+(n+1) / 2,2+(n+1) / 2, \ldots, 2^{k-1}-1+(n+1) / 2\right\}$.

Поскольку семейство $\left\{[\mathbf{v}]: \mathbf{v} \in \mathbf{H}_{k-1}, w(\mathbf{v})=3\right\}$ подмножеств множества $\left\{1+(n+1) / 2,2+(n+1) / 2, \ldots, 2^{k-1}-1+(n+1) / 2\right\}$ является системой троек Штейнера, существует вектор $\mathbf{v} \in \mathbf{H}_{k-1}$ такой, что $w(\mathbf{v})=3$ и $|[\mathbf{c}] \cap[\mathbf{v}]|=2$. Выберем вектор $\mathbf{c}^{\prime} \in \mathbf{H}_{k}+\mathbf{e}_{j}$ так, что $\left|\left[\mathbf{c}^{\prime}\right] \cap[\mathbf{v}]\right|=1$ и $\left[\mathbf{c}^{\prime}\right] \cap[\mathbf{c}]=\varnothing$. Поскольку $\left|\left[\mathbf{c}^{\prime}\right] \cap[\mathbf{v}]\right|=1$ и каждая координата из координатного множества $\{1,2, \ldots, n\}$ принадлежит ровно $(n-1) / 2$ носителям векторов веса 3 из множества $\mathbf{H}_{k}+\mathbf{e}_{j}$, в силу предложения 3 существует вектор $\mathbf{c}^{\prime} \in \mathbf{H}_{k}+\mathbf{e}_{j}, w\left(\mathbf{c}^{\prime}\right)=3$ такой, что $\left[\mathbf{c}^{\prime}\right] \cap[\mathbf{c}]=\varnothing$. Очевидно, что при таком выборе векторов $\mathbf{c}^{\prime}$ и $\mathbf{v}$ справедливо равенство (3).

Далее, допустим, что $i, j \in X$ и вектор $\mathbf{c} \in \mathbf{H}_{k}+\mathbf{e}_{i}$ таков, что $|[\mathbf{c}] \cap X|=3$. Выбираем вектор $\mathbf{c}^{\prime}$ так, что $\left|\left[\mathbf{c}^{\prime}\right] \cap X\right|=2$ и $\left|\left[\mathbf{c}^{\prime}\right] \cap[\mathbf{c}]\right|=1$. В силу предложения 3 такой выбор возможен. Вектор $\mathbf{v}$ выбирается так, что $\left|\left[\mathbf{c}^{\prime}\right] \cap[\mathbf{v}]\right|=2$. Очевидно, что и в этом случае при таком выборе векторов $\mathbf{c}^{\prime}$ и $\mathbf{v}$ справедливо равенство (3).

Далее, допустим, что $i, j \in Y$ и вектор $\mathbf{c} \in \mathbf{H}_{k}+\mathbf{e}_{i}$ таков, что $|[\mathbf{c}] \cap X|=1$. Тогда выбираем векторы $\mathbf{c}^{\prime}$ и $\mathbf{v}$ так же, как в предыдущем случае. При других вариантах выбора координат $i$ и $j$ предложение 4 доказывается аналогично.

Предложение 4 доказано.

Пусть векторы, принадлежащие коду $\mathbf{H}_{k}$, линейно упорядочены так, что первым вектором является нулевой вектор, а последним - вектор $\mathbf{c}_{0} \in \mathbf{H}_{k}, w\left(\mathbf{c}_{0}\right)=3$, и пусть векторы, принадлежащие смежному классу $\mathbf{H}_{k}+\mathbf{e}_{i}, i \in\left\{1,2, \ldots, n=2^{k}-1\right\}$, линейно упорядочены так, что некоторый вектор $\mathbf{c}_{i} \in \mathbf{H}_{k}+\mathbf{e}_{i}, w\left(\mathbf{c}_{i}\right)=3$, является последним, а вектор $\left(\mathbf{c}_{i}+\left(\mathbf{0} \mid \mathbf{v}_{i}\right)\right)$ является первым (здесь $\mathbf{0}-$ нулевой вектор длины $(n+1) / 2=2^{k-1}$, $\left.\mathbf{v}_{i} \in \mathbf{H}_{k-1}\right)$. Кроме того, пусть для выбранных векторов $\mathbf{c}_{0}, \mathbf{c}_{1}, \mathbf{c}_{2} \ldots, \mathbf{c}_{n}$ и $\mathbf{v}_{1}, \mathbf{v}_{2}, \ldots, \mathbf{v}_{n}$ справедливо равенство

$$
d\left(\mathbf{c}_{i-1},\left(\mathbf{c}_{i}+\left(\mathbf{0} \mid \mathbf{v}_{i}\right)\right)\right)=3,
$$

где $i \in\left\{1,2, \ldots, n=2^{k}-1\right\}$. Множество $\mathbf{F}_{2}^{n}$, упорядоченное описанным выше способом, обозначим через $\mathbf{L}_{n}$.

Теорема 2. При $k=3,4, \ldots$ и $n=2^{k}-1$ упорядоченное множество $\mathbf{L}_{n}$ является ииклическим двоичным кодом Грея размерности п с расстоянием 3. 
Доказательство. В силу теоремы 1 векторы, принадлежащие коду $\mathbf{H}_{k}$, возможно линейно упорядочить так, что первым вектором будет нулевой вектор, а последним - вектор $\mathbf{c}_{0} \in \mathbf{H}_{k}, w\left(\mathbf{c}_{0}\right)=3$. В силу предложения 3 возможно выбрать векторы $\mathbf{c}_{1}, \mathbf{c}_{2} \ldots, \mathbf{c}_{n}$ и $\mathbf{v}_{1}, \mathbf{v}_{2}, \ldots, \mathbf{v}_{n}$ так, что будет выполняться равенство (4). В силу теоремы 1 векторы, принадлежащие смежному классу $\mathbf{H}_{k}+\mathbf{e}_{i}, i \in\left\{1,2, \ldots, n=2^{k}-1\right\}$, могут быть упорядочены так, что первым вектором будет вектор $\mathbf{c}_{i}+\left(\mathbf{0} \mid \mathbf{v}_{i}\right)$, а последним - вектор $\mathbf{c}_{i} \in \mathbf{H}_{k}+\mathbf{e}_{i}$, $w\left(\mathbf{c}_{i}\right)=3$. Поскольку последним является вектор веса 3, а первым - нулевой вектор, упорядоченное множество $\mathbf{L}_{n}$ является циклическим двоичным кодом Грея размерности $n$ с расстоянием 3 . Теорема 2 доказана.

\section{4. Нелинейные совершенные коды}

Ниже приведены нелинейные совершенные двоичные коды и конструктивно показано, что графы минимальных расстояний этих кодов содержат гамильтонов цикл.

Пусть подмножество $\mathbf{D}_{4} \subset \mathbf{F}_{2}^{15}$ получено из кода Хемминга $\mathbf{H}_{4}$ свитчингом или сдвигом одной компоненты этого кода [5]. Известно, что $\mathbf{D}_{4}$ является нелинейным совершенным двоичным кодом длины $n=15$. При помощи компьютера несложно проверить, что граф минимальных расстояний кода $\mathbf{D}_{4}$ является гамильтоновым.

Далее воспользуемся uv-конструкцией $[4,5]$. При $k>4$ положим

$$
\mathbf{D}_{k}=\left\{(\mathbf{u}|p(\mathbf{u})| \mathbf{u}+\mathbf{v}): \mathbf{u} \in \mathbf{F}_{2}^{(n-1) / 2},(n-1) / 2=2^{k-1}-1, \mathbf{v} \in \mathbf{D}_{k-1}\right\} .
$$

Множество $\mathbf{D}_{k}$ является совершенным двоичным кодом длины $n=2^{k}-1$. Совершенный код $\mathbf{D}_{k}$ при $k>4$ является нелинейным в силу индуктивного построения и того, что код $\mathbf{D}_{4}$ является нелинейным.

Непосредственно из определения следует, что все совершенные двоичные коды с параметрами кодов Хемминга длины $n$ имеют одинаковый весовой спектр и, в частности, если вектор веса 0 принадлежит коду, то вектор веса $n$ также принадлежит коду. Следовательно, если вектор с принадлежит коду, то и вектор, комплементарный вектору с, так же принадлежит коду. Комплементарным векторам соответствуют антиподальные вершины булева куба.

Теорема 3. При $k \geqslant 4$ граф минимальных расстояний нелинейного соверменного двоичного кода $\mathbf{D}_{k}$ является гамильтоновым.

Доказательство. Теорема доказывается индукцией по $k$. Как было отмечено выше, граф $G\left(\mathbf{D}_{4}\right)$ является гамильтоновым. Покажем, что из индуктивного предположения о гамильтоновости графа $G\left(\mathbf{D}_{k-1}\right)$ следует гамильтоновость графа $G\left(\mathbf{D}_{k}\right)$.

Переписывая формулу (5) с учетом введенных ранее обозначений, получаем равенство

$$
\mathbf{D}_{k}=\bigcup_{\mathbf{v} \in \mathbf{D}_{k-1}}\left(\mathbf{R}_{k}+(\mathbf{0} \mid \mathbf{v})\right),
$$

где $\mathbf{0}$ - нулевой вектор длины $(n+1) / 2=2^{k-1}$. В коде $\mathbf{D}_{k}$ рассмотрим подкоды $\bigcup_{\mathbf{v} \in \mathbf{D}_{k-1}}(\mathbf{0} \mid \mathbf{v})$ и $\bigcup_{\mathbf{v} \in \mathbf{D}_{k-1}}(\mathbf{1}+(\mathbf{0} \mid \mathbf{v}))$, где $\mathbf{1}-$ единичный вектор длины $n$ (то есть вектор, все компоненты которого равны 1). Очевидно, что выбранные подкоды являются комплементарными, то есть содержат комплементарные векторы. По индуктивному предположению, в подкоде $\bigcup_{\mathbf{v} \in \mathbf{D}_{k-1}}(\mathbf{0} \mid \mathbf{v})$ существует гамильтонов цикл. Выберем в подкоде $\bigcup_{\mathbf{v} \in \mathbf{D}_{k-1}}(\mathbf{1}+(\mathbf{0} \mid \mathbf{v}))$ гамильтонов цикл, комплементарный циклу в подкоде $\bigcup_{\mathbf{v} \in \mathbf{D}_{k-1}}(\mathbf{0} \mid \mathbf{v})$. 
Разобьем выбранные гамильтоновы циклы на совершенные паросочетания. В силу предложения 1 граф $G\left(\mathbf{R}_{k}\right)$ изоморфен булеву кубу. Следовательно, в графе $G\left(\mathbf{R}_{k}\right)$ существует гамильтонов путь, в котором первой вершиной является нулевой вектор, а последней вершиной - единичный вектор. Комплементарные совершенные паросочетания в подкодах $\bigcup_{\mathbf{v} \in \mathbf{D}_{k-1}}(\mathbf{0} \mid \mathbf{v})$ и $\bigcup_{\mathbf{v} \in \mathbf{D}_{k-1}}(\mathbf{1}+(\mathbf{0} \mid \mathbf{v}))$ и гамильтоновы пути в $G\left(\mathbf{R}_{k}\right)$ с первой нулевой вершиной и последней единичной образуют гамильтонов цикл в графе $G\left(\mathbf{D}_{k}\right)$. Теорема 3 доказана.

Допустим, что $\mathbf{C}$ - совершенный двоичный код длины $n$ и граф минимальных расстояний кода $\mathbf{C}$ содержит гамильтонов цикл. Тогда с помощью uv-конструкции можно построить совершенный двоичный код $\mathbf{C}^{\prime}$ длины $2 n+1$ такой, что граф минимальных расстояний кода $\mathbf{C}^{\prime}$ также будет содержать гамильтонов цикл.

Нами приведен только один пример совершенного двоичного кода длины $n=15$ с гамильтоновым графом минимальных расстояний. Вполне вероятно, что существуют и другие совершенные двоичные коды длины $n=15$ с гамильтоновыми графами минимальных расстояний. Вопрос о перечислении всех попарно неэквивалентных совершенных двоичных кодов длины $n=15$ остается открытым [5].

\section{Список литературы}

1. Savage C., A survey of combinatorial Gray codes. SIAM Review (1997) 39, №4, 605-629.

2. Пережогин А. Л., О циклических перечислениях двоичных наборов через заданные расстояния. Дискретный анализ и исследование операщий. Сер. 1 (1999) 6, №2, 62-69.

3. Мак-Вильямс Ф. Дж., Слоэн Н. Дж. А., Теория кодов, исправляющих ошибки. Связь, Москва, 1979.

4. Васильев Ю. Л., О негрупповых плотно упакованных кодах. Проблемы кибернетики (1962) 8, 337-339.

5. Романов А. М., Обзор методов построения нелинейных совершенных двоичных кодов. Дискретный анализ и исследование операџฺй. Сер. 1 (2006) 13, №4, 60-88.

Статья поступила 5.05.2008.

Переработанный вариант поступил 5.05.2009. 